SLAC-PUB-10244

Revised

December 2003

\title{
Quantized Cosmology*
}

\author{
Marvin Weinstein and Ratin Akhoury ${ }^{\dagger}$ \\ Stanford Linear Accelerator Center, Stanford University, Stanford, California 94309
}

\begin{abstract}
This paper discusses the problem of inflation in the context of Friedmann-Robertson-Walker Cosmology. We show how, after a simple change of variables, one can quantize the problem in a way which parallels the classical discussion. The result is that two of the Einstein equations arise as exact equations of motion and one of the usual Einstein equations (suitably quantized) survives as a constraint equation to be imposed on the space of physical states. However, the Friedmann equation, which is also a constraint equation and which is the basis of the WheelerDeWitt equation, acquires a welcome quantum correction that becomes significant for small scale factors. We discuss the extension of this result to a full quantum mechanical derivation of the anisotropy $(\delta \rho / \rho)$ in the cosmic microwave background radiation, and the possibility that the extra term in the Friedmann equation could have observable consequences. Finally, we suggest interesting ways in which these techniques can be generalized to cast light on the question of chaotic or eternal inflation. In particular, we suggest one can put an experimental lower bound on the distance to a universe with a scale factor very different from our own, by looking at its effects on our CMB radiation.
\end{abstract}

PACS numbers: F06.60.Ds, 98.80.Hw, 98.80.Cq

\section{INTRODUCTION}

The COBE[1] and WMAP[2][3][4] measurements of the anisotropy in the cosmic microwave background(CMB) radiation agree remarkably well the predictions of slow-roll inflation[5]. This agreement provides a strong reason to believe that the paradigm for computing these fluctuations[6] in $\delta \rho / \rho$ is correct. Perhaps the most striking feature of this result is that, according to the paradigm, they represent an imprinting of the structure of the quantum state of the field theory, at the time inflation begins, onto the electromagnetic radiation that comes to us from the surface of last scattering.

Unfortunately derivations of this effect which appear in the literature mix classical and quantum ideas. Thus, it is hard to pin down the degree to which these results would change if one had a totally quantum mechanical derivation. This paper is devoted to filling this gap. We will show that it is possible to work in a fixed coordinate system and canonically quantize the theory of the Friedmann-Robertson-Walker (FRW) metric,

$$
d s^{2}=-d t^{2}+a(t)^{2} d \vec{x} \cdot d \vec{x},
$$

and the spatially constant part of the inflaton field, $\Phi(t)$, in a straightforward way. The result of this computation is to obtain Heisenberg equations of motion which, starting in a suitably defined coherent state, go over to the conventional Einstein equations for large values of $\langle a(t)\rangle$. This is not true, however, for all values of the scale factor. For small scale factor we find that the Friedman equation gets an important quantum contribution. For even smaller values of the scale factor we find the system goes completely quantum mechanical and one does not expect to see any remnant of the classical solution. In the latter sections of this paper we discuss the way to extend this derivation to compute possible experimental consequences of this extra term.

Derivations of $\delta \rho / \rho$ invoke classical arguments in two ways. These derivations all begin by treating the scale factor $a(t)$ as a classical time-dependent background field. One ignores everything but the spatially constant part of the inflaton field and studies the physics of the classical action

$$
\mathcal{S}=\mathbf{V} \int d t \sqrt{-g}\left[\frac{R(t)}{2 \kappa^{2}}+\frac{1}{2} \frac{d \Phi(t)}{d t}^{2}-V(\Phi(t))\right] .
$$

\footnotetext{
* This work was supported by the U. S. DOE, Contract No. DE-AC03-76SF00515.

$\dagger$ On sabbatical leave from Dept. of Physics, University of Michigan, Ann Arbor, MI 48109-1120
} 
(This implicitly assumes that the constant part of the inflaton field $\Phi(t)$ can be written as a c-number term plus a quantum term. Then the quantum part is ignored.)

The second appearance of classical ideas occurs when one adds back spatially varying fluctuations in the Newtonian potential and the inflaton field as quantum operators and perturbatively studies the behavior of these fields in the background of the classical solution. It is customary, at an appropriate point in the discussion of how these fields behave, to employ the phrase "and then the field goes classical". This much less important introduction of classical ideas is used to convert the quantum computation of the two-point correlation function for the density operator to an ensemble average of gaussian fluctuations. In reality, this statement is just a way of avoiding any discussion of the physics of squeezed states and quantum non-demolition variables. While we do not discuss this issue in this paper, we will return to it in a longer, more pedagogical paper, which is in preparation. This longer paper will show how to extend the results presented here to a full quantum treatment of $\delta \rho / \rho$. The point we wish to emphasize at this juncture is that a full quantum treatment of the spatially constant part of the problem, appropriately extended to include the spatially varying modes of the fields to second order, provides a complete quantum picture of all of the physics which can be experimentally tested in the foreseeable future.

\section{THE CLASSICAL PROBLEM}

Simplifying the usual derivations is easily accomplished if one observes that experimentally we are dealing with a spatially flat universe and so it is perfectly adequate to formulate the problem in what general relativists call a fixed gauge; i.e. a definite coordinate system. In the discussion which follows, we take this to be co-moving coordinates in which the metric takes the general form shown in Eq.1.

As already noted, if we restrict attention to the classical problem of a scalar field in an FRW cosmology, the action reduces to the form shown in Eq.2, where $\mathbf{V}$ is the volume of the region in which the theory is being defined, $\sqrt{-g}=a(t)^{3}$ and the scalar curvature is given by

$$
R(t)=\frac{3}{\kappa^{2}} a(t) \frac{d a(t)^{2}}{d t}+\frac{3}{\kappa^{2}} a(t)^{2} \frac{d^{2} a(t)}{d t^{2}} .
$$

(Clearly, when we generalize to the computation of $\delta \rho / \rho$, the volume, $\mathbf{V}$, must be taken to be larger than the horizon volume at the time of inflation in order to avoid edge effects.)

Substituting these expressions into Eq.2 and integrating by parts, to eliminate the term with $d^{2} a(t) / d t^{2}$, we obtain

$$
\mathcal{S}=\mathbf{V}\left[-\frac{3}{\kappa^{2}} a(t)\left(\frac{d a(t)}{d t}\right)^{2}+\frac{1}{2} a(t)^{3}\left(\frac{d \Phi(t)}{d t}\right)^{2}-a(t)^{3} V(\Phi(t))\right]
$$

Next, in order to simplify the analysis of the quantum version of this problem, we make the change of variables $u(t)^{2}=a(t)^{3}$, which leads to the action

$$
\mathcal{S}=\mathbf{V}\left[-\frac{4}{3 \kappa^{2}}\left(\frac{d u(t)}{d t}\right)^{2}+\frac{1}{2} u(t)^{2}\left(\frac{d \Phi(t)}{d t}\right)^{2}-u(t)^{2} V(\Phi(t))\right] .
$$

This change of variables merely simplifies the classical discussion, however it has a greater significance for the quantized theory. This is because we can choose $-\infty \leq u \leq \infty$, whereas the only physically allowable range for $a$ is $0 \leq a \leq \infty$. It is only for the space of square-integrable functions on the interval $-\infty \leq u \leq \infty$ that the Heisenberg equations of motion can be obtained by canonical manipulations.

\section{CLASSICAL EULER-LAGRANGE EQUATIONS}

There are only two Euler-Lagrange equations for this system:

$$
\frac{8}{3 \kappa^{2}} \frac{d^{2} u(t)}{d t^{2}}+2 u(t)\left(\frac{1}{2}\left(\frac{d \Phi(t)}{d t}\right)^{2}-V(\Phi(t))\right)=0 \quad \text { and } \quad-u(t)^{2}\left(\frac{d^{2} \Phi(t)}{d t^{2}}+3 \mathcal{H}(t) \frac{d \Phi(t)}{d t}+\frac{d V(\Phi)}{d \Phi(t)}\right)=0 ;
$$

where the Hubble parameter, $\mathcal{H}$, is defined as

$$
\mathcal{H}=\frac{1}{a(t)} \frac{d a(t)}{d t}=\frac{2}{3 u(t)} \frac{d u(t)}{d t} .
$$


Thus, by quantizing in this fixed gauge, we fail to obtain the full set of Einstein equations. The missing equations are the Friedmann equation and its time derivative

$$
\mathcal{H}(t)^{2}=\frac{\kappa^{2}}{3}\left(\frac{1}{2}\left(\frac{d \Phi(t)}{d t}\right)^{2}+V(\Phi(t))\right) \quad \text { and } \quad \frac{d \mathcal{H}(t)}{d t}=-\frac{\kappa^{2}}{2}\left(\frac{d \Phi(t)}{d t}\right)^{2}
$$

A sophisticated way of explaining why we fail to obtain these equations is to note that fixing the form of the metric to be that given in Eq.1, we have lost the freedom to vary the lapse and shift functions. But this is what we must do to obtain the missing equations from a Lagrangian formulation. This predicament is not unique to gravity; it occurs in ordinary electrodynamics if one chooses $A_{0}=0$ gauge. As is well known, in this gauge we obtain all of the Maxwell equations except Coulomb's law, $\vec{\nabla} \cdot \vec{E}-\rho=0$, as exact equations of motion. However, it follows from the equations we do have, that Coulomb's law commutes with the evolution, i.e. if we set it equal to zero it remains zero. Hence, in this gauge, while there are many solutions to the equations of motion, we can select the ones we choose to call physical by imposing an extra time-independent constraint.

The situation with the Friedmann equation and its time derivative is analogous to the situation in electrodynamics. We will now show that while Eqs.8, are not equations of motion, if they are imposed at any one time, then they will continue to be true at all later times. (In other words they are constraint equations.)

To prove these constraints are preserved by the equation of motion we begin by differentiating $\mathcal{H}$ with respect to $t$ to obtain

$$
\frac{d^{2} u(t)}{d t^{2}}=\frac{3 u(t)}{2}\left(\frac{d \mathcal{H}(t)}{d t}+\frac{3}{2} \mathcal{H}(t)^{2}\right)
$$

Substituting this into Eq.6 and rearranging terms we obtain

$$
\frac{2 u(t)}{\kappa^{2}}\left(2 \frac{d \mathcal{H}(t)}{d t}+3 \mathcal{H}(t)^{2}+\kappa^{2}\left(\frac{d \Phi(t)}{d t}\right)^{2}-\kappa^{2}\left(\frac{1}{2}\left(\frac{d \Phi(t)}{d t}\right)^{2}+V(\Phi(t))\right)\right)=0
$$

which can be immediately rewritten in the form

$$
\frac{2 u(t)}{\kappa^{2}}\left[\left(2 \frac{d \mathcal{H}(t)}{d t}+\kappa^{2}\left(\frac{d \Phi(t)}{d t}\right)^{2}\right)+3\left(\mathcal{H}(t)^{2}-\frac{\kappa^{2}}{3}\left(\frac{1}{2}\left(\frac{d \Phi(t)}{d t}\right)^{2}+V(\Phi(t))\right)\right)\right]=0
$$

If we define

$$
\mathbf{G}=\mathcal{H}(t)^{2}-\frac{\kappa^{2}}{3}\left(\frac{1}{2}\left(\frac{d \Phi(t)}{d t}\right)^{2}+V(\Phi(t))\right)
$$

then the equation of motion for $\Phi(t)$ implies

$$
\frac{d \mathbf{G}}{d t}=2 \mathcal{H}(t) \frac{d \mathcal{H}(t)}{d t}+\kappa^{2} \mathcal{H}(t)\left(\frac{d \Phi(t)}{d t}\right)^{2}=2 \mathcal{H}(t)\left(\frac{d \mathcal{H}(t)}{d t}+\frac{\kappa^{2}}{2}\left(\frac{d \Phi(t)}{d t}\right)^{2}\right)
$$

The missing Einstein equations are equivalent to requiring that both $\mathbf{G}$ and $d \mathbf{G} / d t$ vanish for all time. Substituting these definitions into Eq.11, we obtain the exact equation of motion

$$
\frac{2 u(t)}{\kappa^{2}}\left(\frac{1}{\mathcal{H}(t)}\left(\frac{d \mathbf{G}}{d t}\right)+3 \mathbf{G}\right)=0
$$

From this equation we see that if, at time $t=t_{0}, \mathbf{G}=0$, then from the exact equation of motion $d \mathbf{G} / d t$ will also vanish. Finally, by taking successive derivatives of Eq.14, we see that all derivatives of $\mathbf{G}$ vanish. In other words, we arrive at the desired result. The Friedmann equation is, in direct analogy to Coulomb's law in $A_{0}=0$ gauge, a constraint which can be imposed at a single time and which will continue to be true at all later times. As we will see, a similar theorem can be proven for the quantum theory; however, the analogy with QED will not be perfect. 


\section{CLASSICAL HAMILTONIAN ANALYSIS}

Before moving on to the quantum theory, let us spend a few moments discussing the Hamiltonian version of the classical theory. We do this to show why it is possible to confuse the Friedmann equation with the Hamiltonian at the classical level .

Following the usual prescription, we vary Eq.4 with respect to $d u / d t$ and $d \Phi / d t$ to obtain

$$
p_{u}=-\mathbf{V} \frac{8}{3 \kappa^{2}} \frac{d u(t)}{d t} \quad ; \quad p_{\Phi}=\mathbf{V} u^{2} \frac{d \Phi(t)}{d t}
$$

We then construct the Hamiltonian

$$
\mathbf{H}=p_{u} \frac{d u(t)}{d t}+p_{\Phi} \frac{d \Phi(t)}{d t}-\mathcal{L}=-\frac{3 \kappa^{2}}{16 \mathbf{V}} p_{u}^{2}+\frac{1}{2 \mathbf{V} u^{2}} p_{\phi}^{2}+\mathbf{V} u^{2} V(\Phi)
$$

An important feature of this Hamiltonian is that due to the minus sign in front of the $p_{\mu}^{2}$ term, it has no minimum. Fortunately, this doesn't matter. To see this, we simply rewrite the Hamiltonian in terms of $d u / d t$ and $d \Phi / d t$. This leads to the expression

$$
\mathbf{H}=\mathbf{V}\left[-\frac{4}{3 \kappa^{2}}\left(\frac{d u(t)}{d t}\right)^{2}+u^{2}\left(\frac{1}{2}\left(\frac{d \Phi(t)}{d t}\right)^{2}+V(\Phi)\right)\right] .
$$

Substituting the definition of $\mathcal{H}$, this becomes

$$
\begin{aligned}
\mathbf{H} & =-\mathbf{V} u^{2}\left[\frac{4}{3 \kappa^{2}} \frac{1}{u^{2}}\left(\frac{d u(t)}{d t}\right)^{2}-\left(\frac{1}{2}\left(\frac{d \Phi(t)}{d t}\right)^{2}+V(\Phi)\right)\right]=-\mathbf{V} u^{2}\left[\frac{3 \mathcal{H}^{2}}{\kappa^{2}}-\left(\frac{1}{2}\left(\frac{d \Phi(t)}{d t}\right)^{2}+V(\Phi)\right)\right] \\
\mathbf{H} & =-\mathbf{V} \frac{3 u^{2}}{\kappa^{2}} \mathbf{G} .
\end{aligned}
$$

This shows that the Hamiltonian, $\mathbf{H}$, is proportional to the constraint, $\mathbf{G}$. It follows that setting $\mathbf{G}=0 \mathrm{means} \mathbf{H}=0$, which tells us that the Hamiltonian vanishes for physical solutions. In other words, if we start a system out at $t=t_{0}$ in a configuration which has zero energy, it will stay at zero energy and never explore the region of arbitrarily negative energy. The identification of the Hamiltonian with the constraint equation is the content of the Wheeler-DeWitt equation.

\section{CANONICAL QUANTIZATION OF THE THEORY}

Starting from the classical Lagrangian, we define the quantum Hamiltonian

$$
\mathbf{H}=-\frac{3 \kappa^{2}}{16 \mathbf{V}} p_{u}^{2}+\frac{1}{2 \mathbf{V} u^{2}} p_{\Phi}^{2}+\mathbf{V} u^{2} V(\Phi)
$$

where the operators $u, \Phi$ and their conjugate momenta have the commutation relations

$$
\left[p_{u}, u\right]=-i \quad ; \quad\left[p_{\Phi}, \Phi\right]=-i
$$

All other commutators vanish. To derive the Heisenberg equations of motion, note that for any operator $\mathbf{O}$, the Heisenberg operator is $O(t)=e^{i \mathbf{H} t} \mathbf{O} e^{-i \mathbf{H} t}$. Commuting $\mathbf{H}$ with the operators $u$ and $\Phi$, we obtain

$$
\begin{aligned}
\frac{d u(t)}{d t} & =i[\mathbf{H}, u]=-\frac{3 \kappa^{2}}{8 \mathbf{V}} p_{u} \\
\frac{d \Phi(t)}{d t} & =i[\mathbf{H}, \Phi]=\frac{1}{u^{2} \mathbf{V}} p_{\Phi} \\
\frac{d^{2} u(t)}{d t^{2}} & =i\left[\mathbf{H}, \frac{d u(t)}{d t}\right]=-\frac{3 \kappa^{2}}{4} u\left[\frac{1}{2}\left(\frac{d \Phi(t)}{d t}\right)^{2}-V(\Phi)\right] \\
\frac{d^{2} \Phi(t)}{d t^{2}} & =\frac{3 \kappa^{2}}{16 \mathbf{V}}\left(\frac{1}{u^{2}} p_{u} \frac{1}{u}+\frac{1}{u} p_{u} \frac{1}{u^{2}}+p_{u} \frac{1}{u^{3}}+\frac{1}{u^{3}} p_{u}\right) p_{\Phi}-\frac{d V(\Phi)}{d \Phi}
\end{aligned}
$$


This shows that the two dynamical equations of the classical theory are also exact operator equations of motion in the quantum theory. What is missing, as in the classical theory, are the constraint equations. In order to find the constraint equations that commute with the Hamiltonian, we begin by rewriting the equation for $\Phi$ in the suggestive form

$$
\frac{d^{2} \Phi(t)}{d t^{2}}+3 \mathcal{H} \frac{d \Phi(t)}{d t}+\frac{d V(\Phi)}{d \Phi}=0
$$

where the quantum version of the Hubble operator $\mathcal{H}$ is perforce

$$
\mathcal{H}=-\frac{\kappa^{2}}{8 \mathbf{V}}\left(p_{u} \frac{1}{u}+\frac{1}{u^{3}} p_{u} u^{2}\right) .
$$

Next we compute its time derivative from the equation

$$
\frac{d \mathcal{H}}{d t}=i[\mathbf{H}, \mathcal{H}]
$$

Finally, to find the quantum version of the conserved constraint operator, $\mathbf{G}$, we follow the classical procedure and write

$$
\frac{d^{2} u(t)}{d t^{2}}=\frac{3 u}{2}\left(\frac{d \mathcal{H}}{d t}+\frac{3}{2} \mathcal{H}^{2}-\frac{9 \kappa^{4}}{128 \mathbf{V}^{2} u^{4}}\right)
$$

The extra term is the quantum correction to the classical formula. It is obtained by explicitly taking the difference between the expression for $d^{2} u / d t^{2}$ and the combination $(3 u / 2)\left(d \mathcal{H} / d t+3 \mathcal{H}^{2} / 2\right)$. (This step involves commutator gymnastics better left to Maple.) Once again, paralleling the classical discussion, we substitute the expression for $d^{2} u / d t^{2}$ into the Heisenberg equation of motion for $u$, obtaining

$$
\frac{3 u}{2}\left(\frac{d \mathcal{H}}{d t}+\frac{3}{2} \mathcal{H}^{2}-\frac{9 \kappa^{4}}{128 \mathbf{V}^{2} u^{4}}\right)+\frac{3 \kappa^{2} u}{4}\left(\left(\frac{d \Phi(t)}{d t}\right)^{2}-\left(\frac{1}{2}\left(\frac{d \Phi(t)}{d t}\right)^{2}+V(\Phi)\right)\right)=0 .
$$

At this point it is tempting to parallel the classical discussion and define the operator

$$
\mathbf{G}=\mathcal{H}^{2}-\frac{\kappa^{2}}{3}\left(\frac{1}{2}\left(\frac{d \Phi(t)}{d t}\right)^{2}+V(\Phi)\right)+\mathbf{Q}
$$

where

$$
\mathbf{Q}=-\frac{3 \kappa^{4}}{64 \mathbf{V}^{2} u^{4}}
$$

and show that if it annihilates a state at any one time, then it annihilates it for all times. We will now show that this can be done, however we will then argue that identifying the kernel of this operator with the space of physical states is incorrect. To proceed with the proof substitute this definion into Eq.26 to obtain the operator equation of motion

$$
\frac{3 u}{4}\left(2 \frac{d \mathcal{H}(t)}{d t}+\kappa^{2}\left(\frac{d \Phi(t)}{d t}\right)^{2}+3 \mathbf{G}\right)=0 .
$$

This is almost what we need to show that the space of physical states, defined to be those which obey the condition $\mathbf{G}(t)|\psi\rangle=0$, is invariant under Hamiltonian evolution. Clearly, we will be able to use Eq.29 to complete the proof if we can show that there exists an operator $\mathbf{A}$ such that

$$
\frac{d \mathbf{G}}{d t}=\mathbf{A}\left(2 \frac{d \mathcal{H}(t)}{d t}+\kappa^{2}\left(\frac{d \Phi(t)}{d t}\right)^{2}\right) .
$$

To find A, explicitly compute

$$
\begin{aligned}
\frac{d \mathbf{G}}{d t} & =i[\mathbf{H}, \mathbf{G}] \\
& =\mathcal{H} \frac{d \mathcal{H}(t)}{d t}+\frac{d \mathcal{H}(t)}{d t} \mathcal{H}+\kappa^{2} \mathcal{H}\left(\frac{d \Phi(t)}{d t}\right)^{2}+[\mathbf{H}, \mathbf{Q}] \\
& =\mathcal{H}\left(2 \frac{d \mathcal{H}(t)}{d t}+\kappa^{2}\left(\frac{d \Phi(t)}{d t}\right)^{2}\right)+\left[\frac{d \mathcal{H}(t)}{d t}, \mathcal{H}\right]+[\mathbf{H}, \mathbf{Q}]
\end{aligned}
$$


and substitute this result into Eq.30. The resulting equation can then be rearranged into the form

$$
(\mathbf{A}-\mathcal{H})\left(2 \frac{d \mathcal{H}(t)}{d t}+\kappa^{2}\left(\frac{d \Phi(t)}{d t}\right)^{2}\right)=[\mathbf{H}, \mathbf{Q}]+\left[\frac{d \mathcal{H}(t)}{d t}, \mathcal{H}\right] .
$$

Solving this equation for $\mathbf{A}$, we obtain

$$
\mathbf{A}=\mathcal{H}+\left([\mathbf{H}, \mathbf{Q}]+\left[\frac{d \mathcal{H}(t)}{d t}, \mathcal{H}\right]\right)\left(2 \frac{d \mathcal{H}(t)}{d t}+\kappa^{2}\left(\frac{d \Phi(t)}{d t}\right)^{2}\right)^{-1}
$$

which allows us to rewrite the Heisenberg equation of motion for $u$ as

$$
\frac{3 u}{4}\left(\frac{1}{\mathbf{A}} \frac{d \mathbf{G}}{d t}+3 \mathbf{G}\right)=0 .
$$

Given that Eq.34 is an exact operator equation of motion, we see that if we could define the space of states by the condition $\mathbf{G}\left(\mathbf{t}_{\mathbf{0}}\right)|\psi\rangle=0$, then Eq.34 proves that this condition will hold for all time. Note, however, that given this definition of $\mathbf{G}$, it follows immediately that

$$
\mathbf{G}(t)=-\frac{\kappa^{2}}{3 \mathbf{V} u(t)^{2}} \mathbf{H}
$$

Thus one is forced to conclude that this definition the space of physical states, implies that they are states for which the Hamiltonian vanishes. Obviously, if we define the physical states in this way we immediately produce a contradiction between the Schroedinger and Heisenberg equations of motion. The resolution of this apparent paradox is that the correct definition of the space of physical states is, $|\Psi\rangle$ is a physical state, if and only if,

$$
\lim _{u(t)^{2} \rightarrow \infty} \mathbf{G}(t)|\Psi\rangle=0 .
$$

This condition is satisfied in any cosmological theory, in which $u(t)$ grows as $t \rightarrow \infty$, for any state $|\Psi\rangle$ such that $\mathbf{H}|\Psi\rangle$ has finite norm. One way of rewriting this condition is that Eq.36 is true for all states $\Psi$ for which

$$
\left\langle\Psi\left|H^{2}\right| \Psi\right\rangle<\infty .
$$

In the paper which follows, we provide an exact solution of the theory in which $V(\Phi)$ is replaced by a constant; i.e., de-Sitter space. There we explicitly show that this form of the constraint equation is the only one consistent with the physics.

Before leaving this issue and moving on to a general discussion of how one recovers the classical theory, it is worth stating that, in fact, any operator of the form

$$
\mathbf{G}_{\alpha}=\mathcal{H}^{2}-\frac{\kappa^{2}}{3}\left(\frac{1}{2}\left(\frac{d \Phi(t)}{d t}\right)+V(\Phi)\right)+\alpha \mathbf{Q}
$$

will satisfy an equation of the form

$$
\frac{3 u}{4}\left(\frac{1}{\mathbf{A}_{\alpha}} \frac{d \mathbf{G}_{\alpha}}{d t}+3 \mathbf{G}_{\alpha}\right)=0
$$

Of course, only for the choice $\alpha=1$, does the condition that $\mathbf{G}_{\alpha}|\Psi\rangle=0$ imply that the Hamiltonian annihilates the state. One might be tempted to suppose that one of these operators could be used to define the space of physical states, since there would no longer be any obvious contradiction with the Heisenberg equations of motion. However, in the paper which follows, we will argue that no normalizeable states satisfying this gauge-conditions exist for any value of $\alpha$. Thus, for all such choices, the condition in Eq.36 is the correct one.

\section{RECOVERING THE CLASSICAL THEORY}

Recovering the classical picture of slow-roll inflation from these equations is straightforward. Since we are working with the Heisenberg equations of motion, all we have to do is assume that we start from a coherent state $|\psi\rangle$, such 
that $\mathbf{G}|\psi\rangle=0$. Furthermore, we assume that $\langle\psi|u| \psi\rangle,\left\langle\psi\left|p_{u}\right| \psi\right\rangle,\langle\psi|\Phi| \psi\rangle$ and $\left\langle\psi\left|p_{\Phi}\right| \psi\right\rangle$ satisfy the initial conditions required for a classical theory of slow-roll inflation. In this case, it makes sense to rewrite the Heisenberg operators as

$$
u(t)=\widehat{u}(t)+\delta u(t) \quad ; \quad \Phi(t)=\widehat{\Phi}(t)+\delta \Phi(t),
$$

where $\hat{u}(t)$ and $\hat{\Phi}(t)$ are c-number functions such that $\langle\psi|u(t)| \psi\rangle=\hat{u}(t)$ and $\langle\psi|\Phi(t)| \psi\rangle=\hat{\Phi}(t)$. Given these assumptions, we wish to show that if these c-number functions satisfy the classical slow-roll equations for inflation, then as a consequence of inflation, the quantum corrections to the Heisenberg equations of motion will be strongly suppressed.

Substituting these definitions into the Heisenberg equation of motion for the operator $u(t)$ in Eq.21 and the form of the equation of motion for the operator $\Phi(t)$ in Eq.22, we see that if the classical functions $\hat{u}(t)$ and $\hat{\Phi}(t)$ satisfy the classical equations for slow-roll inflation, the c-number terms all cancel, and one is left with equations for the operators $\delta u(t)$ and $\delta \Phi(t)$. At first glance, solving these equations seems difficult; however, the situation improves significantly if we look at the constraint equations

$$
\left[\mathcal{H}^{2}-\frac{\kappa^{2}}{3}\left(\frac{1}{2}(d \Phi(t) / d t)^{2}+V(\Phi(t))\right)+\mathbf{Q}(t)\right]|\psi\rangle=0, \quad ; \quad\left[d \mathcal{H} / d t+\frac{\kappa^{2}}{2}(d \Phi(t) / d t)^{2}\right]|\psi\rangle=0
$$

In the rest of this section we will ignore the operator $\mathbf{Q}(t)$, since it is proportional to $\widehat{u}(t)^{-4}$, which we expect to be small in the inflationary and FRW eras.

The second equation says that in the sector of physical states we can replace the operator $(d \Phi(t) / d t)^{2}$ by $-2(d \mathcal{H} / d t) / \kappa^{2}$. Substituting this in the first constraint yields the equation

$$
\left[3 \mathcal{H}(t)^{2}+\frac{d \mathcal{H}(t)}{d t}-\kappa^{2} V(\Phi(t))\right]|\psi\rangle=0
$$

Substituting Eq.40 into the definition of $\mathcal{H}$, we get

$$
\mathcal{H}(t)=\widehat{\mathcal{H}}(t)+\delta \mathcal{H}(t)
$$

where the form one obtains for $\delta \mathcal{H}(t)$ would seem to imply that the operator shrinks rapidly during the inflationary era because of the inverse power of $\widehat{u}(t)$ appearing in its definition. Nevertheless, it behooves us to check that the operators $\delta u(t)$, etc. do not behave badly. Substituting Eq.43 into the constraint equation and using $\Phi(t)=\widehat{\Phi}(t)+\delta \Phi(t)$, cancelling out the contributions of the c-number functions and keeping terms of first order in $\delta \mathcal{H}$ and $\delta \Phi(t)$, we obtain

$$
\left[6 \widehat{\mathcal{H}} \delta \mathcal{H}-\kappa^{2}\left(\frac{d V(\widehat{\Phi})}{d \Phi}\right) \delta \Phi(t)\right]|\psi\rangle=0
$$

Taking the expectation value of this equation in the state $|\psi\rangle$, noting that by assumption $\langle\psi|\delta \Phi(t)| \psi\rangle=0$, it follows that

$$
6 \widehat{\mathcal{H}}(t)\langle\psi|\delta \mathcal{H}(t)| \psi\rangle+\frac{d\langle\psi|\delta \mathcal{H}(t)| \psi\rangle}{d t}=0
$$

The solution to this equation is

$$
\left\langle\psi\left|\delta \mathcal{H}\left(t_{f}\right)\right| \psi\right\rangle=e^{-6 \int_{t_{i}}^{t_{f}} d t \widehat{\mathcal{H}}(t)}\left\langle\psi\left|\delta \mathcal{H}\left(t_{i}\right)\right| \psi\right\rangle
$$

If we recall that the classical function $\mathcal{H}(t)$ is just $d(\ln (a(t))) / d t$, we see that the integral in Eq.46 is just the number of $e$-foldings during inflation. Thus, the contribution of the operator $\delta \mathcal{H}$ is strongly suppressed.

\section{HOW BIG ARE THE CORRECTIONS?}

We now wish to address the question of whether it makes sense to ignore the operator $\mathbf{Q}=-3 \kappa^{4} / 64 \mathbf{V}^{2} u^{4}$ at the onset of inflation. Obviously, the issue boils down to how large this term is relative to the operator $\kappa^{2} V(\Phi) / 3$. To establish this ratio, we must first specify the value of the quantization volume $\mathbf{V}$. Clearly, there is no upper limit for the value one can choose for $\mathbf{V}$. There is, however, a lower limit, since $\mathbf{V}$ must be chosen larger than the horizon volume at the time of inflation to avoid boundary effects which are not seen in the WMAP data. Thus, $\mathbf{V}>1 / \mathcal{H}_{I}^{3}$, where $\mathcal{H}_{I}$ is the value of the Hubble parameter at the onset of inflation. 
To estimate the size of $\mathcal{H}_{I}$, if the classical approximation dominates, we use the classical version of the Friedmann equation. This equation tells us that

$$
\mathcal{H}_{I}^{2} \approx \frac{\kappa^{2}}{3} V(\Phi)
$$

Substituting this into the expression for $\mathbf{Q}$ we obtain that

$$
\begin{aligned}
\mathbf{Q} & =-\frac{3 \kappa^{4}}{64} \mathcal{H}_{I}^{6} \frac{1}{u^{4}} \\
& =-\frac{\kappa^{10}}{576} V(\Phi)^{3} \frac{1}{u^{4}}
\end{aligned}
$$

which is to be compared to $\kappa^{2} V(\Phi) / 3$. Thus the statement that $\mathbf{Q}$ can be ignored at the onset of inflation is equivalent to

$$
\frac{\kappa^{2}}{3} V(\Phi) \gg \frac{\kappa^{10}}{576} V(\Phi)^{3} \frac{1}{u^{4}}
$$

It is convenient to multiply this equation by a factor of $\kappa^{2}$ to obtain

$$
\kappa^{4} V(\Phi) \gg \frac{1}{192}\left(\kappa^{4} V(\Phi)\right)^{3} \frac{1}{u^{4}},
$$

or equivalently

$$
\frac{1}{192}\left(\kappa^{4} V(\Phi)\right)^{2} \frac{1}{u^{4}} \ll 1
$$

At this point we note that the product $\kappa^{4} V(\Phi)$ is usually constrained to be less than or on the order of $10^{-6}$. Thus, if $u(t)$ is chosen to be the order of unity at the time inflation starts, the effects of $\mathbf{Q}$ will be negligible. Note however that $1 / u(t)^{4}=1 / a(t)^{6}$, so one cannot extrapolate very many $e$-foldings back from the starting point before quantum corrections become important.

\section{STRAIGHTFORWARD EXTENSIONS}

In order to extend this model to a complete treatment of the CMB anisotropy, one has both to add an extra field $\chi(t, x)$ to the metric, in order to model Newtonian fluctuations, and to put the the spatially varying part to the $\Phi$ field back into the action. In other words, the metric is taken to have the form

$$
d s^{2}=-(1+2 \epsilon \chi(t, \vec{x})) d t^{2}+a(t)^{2}(1-2 \epsilon \chi(t, \vec{x})) d \vec{x}^{2}
$$

and the action is taken to be

$$
\mathcal{S}=\int d^{4} x \sqrt{-g}\left[\frac{R(g)}{2 \kappa^{2}}+\frac{1}{1+2 \epsilon \chi(t, \vec{x})}\left(\frac{d \Phi(t)}{d t}+\frac{\epsilon d \phi(t, \vec{x})}{d t}\right)^{2}-\frac{\epsilon^{2} \vec{\nabla} \phi(t, \vec{x}) \cdot \vec{\nabla} \phi(t, \vec{x})}{a(t)^{2}(1-2 \epsilon \chi(t, \vec{x}))}-V(\Phi(t)+\epsilon \phi(t, \vec{x}))\right]
$$

If we now expand this formula up to order $\epsilon^{2}$ we see that: the $\epsilon^{0}$ term is the problem we have been considering; the $\epsilon^{1}$ term vanishes due to the equations of motion; the remaining terms are quadratic in the fields $\chi(t, x)$ and $\phi(t, x)$. Thus, if we start in the coherent state discussed in the previous sections, $\chi(t, x)$ and $\phi(t, x)$ are simply free fields evolving in a time-dependent background and their Heisenberg equations of motion can be solved exactly.

This analysis allows one to completely reproduce the usual computations for $\delta \rho / \rho$. (A complete treatment of this will appear in a forthcoming pedagogical paper.) In other words, this effective theory is capable of reproducing the theory of all CMB measurements within a quantum framework in which the long wavelength part of the gravitational field satisfies the exact Einstein equations, while the shorter wavelengths are treated perturbatively. The small size of the CMB fluctuations tells us this is a reasonable approach.

This extension of the model gives us a canonical Hamiltonian picture of the evolution of the theory. This means that the back-reaction caused by the changes in $\chi(t, x)$ and $\phi(t, x)$ are completely specified. Clearly, it is important to ask if these back-reaction effects, or the effects $\mathbf{Q}$ causes on the evolution of the system, leave an observable imprint on the CMB fluctuation spectrum. 


\section{LESS OBVIOUS EXTENSIONS}

There are two less obvious but very interesting directions in which one can extend this work.

The first is to reintroduce some very long wavelength modes of $\chi(t, x)$ and $\phi(t, x)$ into the part of the Lagrangian that we treat exactly. To be specific, we can expand the field $\chi(t, x)$ and $\phi(t, x)$ in some sort of wavelet basis, for which the low-lying wavelets represent changes over fractions of the horizon scale; i.e.

$$
\chi(t, x)=\sum_{j=1}^{N} b_{j}(t) w_{j}(x)+\epsilon \chi(t, x)^{\prime} \quad ; \quad \phi(t, x)=\sum_{j=1}^{N} c_{j}(t) w_{j}(x)+\epsilon \phi(t, x)^{\prime} .
$$

Next, we can plug this expansion into the action and expand it to second order in $\epsilon$.

In contrast to the previous case, the order $\epsilon^{0}$ part of the action will now be the theory of $2 N$ non-linearly coupled variables. Clearly, we should be able to parallel the discussion given in this paper and proceed to: first, derive the canonical Hamiltonian, then derive the Heisenberg equations of motion for the system, which will not be the full set of Einstein equations, and, finally, construct the proper constraint operators to fill out the full set of equations of motion. The resulting theory should be equivalent to a theory in which we have finite size boxes with independent scale factors that are weakly coupled to one another. Since the usual CMB results imply that fluctuations on all wavelengths are small, it must be true that in this version of the theory the scale factors in neighboring boxes (or pixels) can't get very far from one another without affecting the CMB fluctuations. We suggest that one way to see how a fully quantized theory of gravity behaves at shorter wavelengths is to pixelize the theory in this way. Then we can study what happens to the quantum problem as we add operators corresponding to higher frequency fluctuations in the quantum fields back into the fully non-linear problem.

Another interesting direction is to pixelize a problem initially quantized in a region extending over several horizon volumes. Since the pixelization to volumes smaller than the scale set by the horizon during inflation certainly leads to coupling terms between the pixels, there will be analogous (presumably weaker) couplings between what will now be neighboring horizon-size pixels. The interesting question here is whether or not the scale factors in neighboring volumes can get very far from one another without causing observable changes in the CMB fluctuation spectrum seen by an observer in any individual volume. In other words, can we - within the context of chaotic or eternal inflation - put experimental limits on how near to us a very different universe from ours can be, without leaving a visible imprint on the CMB radiation in our universe? If such limits can be found, we will have found a way to see the unseeable.

\section{SUMMARY}

We have shown how to fully quantize the theory of inflation and the computation of $\delta \rho / \rho$ by working in a fixed gauge (i.e. co-moving coordinates) and making a simple change of variables. Our focus was on the formulation of the part of the problem that involved the spatially constant fields. As we demonstrated, in both the classical and quantum theory, working in a fixed gauge yields only two of the four relevant Einstein equations as equations of motion. In the classical theory we showed that the Friedmann equation and its time derivative must be treated as constraints whose constancy in time requires a proof. This proof followed from the two equations of motion we did have. Next, we showed that, in the quantum version of the theory, the same two Einstein equations appear as operator equations of motion. We then argue that the simplest constraint equation, which corresponds to the Wheeler-DeWitt equation, cannot be used to define the space of physical states. We then show that there exist other possible choices for constraint operators, but choosing them automatically makes the constraint equation different from the Wheeler-DeWitt equation. From this it follows that identifying the Friedmann equation with the condition that the Hamiltonian annihilates physical states is always incorrect. In the paper which follows this one we show explicitly how all of this plays out for the case of de-Sitter space. 


\section{ACKNOWLEDGEMENTS}

MW would like to express his gratitude to J. D. Bjorken for dragging him, kicking and screaming, into looking at this fascinating problem.

[1] J. C. Mather et al., Astrophys. J. 354, L37 (1990).

[2] H. V. Peiris et al., Astrophys. J. Suppl. 148, 213 (2003) [arXiv:astro-ph/0302225].

[3] E. Komatsu et al., Astrophys. J. Suppl. 148, 119 (2003) [arXiv:astro-ph/0302223].

[4] M. R. Nolta et al., arXiv:astro-ph/0305097.

[5] For the original model of inflation which did not really work see A. H. Guth, Phys. Rev. D 23, 347356(1981). The current paradigm of slow-roll inflation which improved the situation was developed separately by Linde and Albrecht and Steinhardt, A. D. Linde, Phys. Lett. B108 389393 (1982) A. Albrecht and P. J. Steinhardt, Phys. Rev. Lett.48, 12201223 (1982).

[6] For references to the general theory of computing cosmological perturbations see V. F. Mukhanov, H. A. Feldman, and R. H. Brandenberger, Phys. Rept. 215, 203-333 (1992), and references cited therein. 